\title{
Adult cervicomedullary pilocytic astrocytoma: A case report
}

\author{
YAODONG ZHAO $^{1}$, LIJIN FENG ${ }^{2}$, QING WEI $^{2}$ and LIANG GAO ${ }^{1}$ \\ Departments of ${ }^{1}$ Neurosurgery and ${ }^{2}$ Pathology, Shanghai 10th People's Hospital, \\ Tongji University, School of Medicine, Shanghai 200072, P.R. China
}

Received October 18, 2014; Accepted September 15, 2015

DOI: $10.3892 /$ etm.2015.2828

\begin{abstract}
Pilocytic astrocytoma (PA) is a rare glioma, which generally occurs in children and young adults. In adult patients, the majority of PA tumors are supratentorial. Due to the low morbidity rate of the disease in adults, PA is frequently misdiagnosed and mistreated. In the present study, this rare disease was successfully treated. The study reported the case of a 48-year-old patient with a cervicomedullary occupying lesion, who complained of numbness and pain of the right limbs that persisted for $>10$ years, with aggravation for 1 month. A magnetic resonance imaging scan showed a sharp cervicomedullary mass extending from the lower medulla to the cervical vertebra C3 level. Intraoperatively, the medulla and upper cervical cord were found to be well-stacked. Immediately after ingression into the spinal cord along the dorsal median sulcus, the tumor mass was detected and had a gray fish-like appearance, moderate blood supply and clear boundary. After intratumoral decompression, total excision was achieved. Postoperative pathological examination confirmed that the tumor was a PA. Following discharge, the patient did not suffer from any symptoms of the lower cranial nerves and was able to walk with limited assistance.
\end{abstract}

\section{Introduction}

Pilocytic astrocytoma (PA) is a rare, slow-growing glioma, which is classified as grade I by the World Health Organization and typically occurs in children and young adults (1). PA is the most common glial neoplasm in children. Only one-third of PA patients are $>18$ years of age and only $17 \%$ are $>30$ years of age, with $50 \%$ of tumors being supratentorial in adults (2). The most common occurrence sites include the region around the third ventricle and the cerebellum. However, the entire neuraxis can be affected, with prevalence in the hypothalamus, thalamus, optical chiasma and nerve, brainstem, cerebellum, cerebral

Correspondence to: Professor Liang Gao, Department of Neurosurgery, Shanghai 10th People's Hospital, Tongji University, School of Medicine, 301 Yanchang Middle Road, Shanghai 200072, P.R. China

E-mail: dm0920@163.com

Key words: pilocytic astrocytoma, cervicomedullary, adult hemispheres and basal ganglia (3-5). Cervicomedullary PA has been rarely reported, and the disease is frequently misdiagnosed due to its rarity. There are no clinical features that are unique to PA. Signs and symptoms usually persist for a number of months, and may differ depending on the size, location and presence of associated hydrocephalus. Patients with PA typically exhibit an extremely high survival rate of $>90 \%$ at 10 years of age (6). The present study reported the case of a 48-year-old patient with cervicomedullary PA that was successfully treated. The study aims to share our clinical experience on this disease. In addition, this patient may be the eldest PA case ever reported.

\section{Case report}

A 48-year-old woman was admitted to the Shanghai 10th People's Hospital (Shanghai, China) in May 2014, complaining of numbness and pain of the right limbs that persisted for $>10$ years and was aggravated for 1 month. No significant medical history was reported, such as any head traumas, and the patient's family history was unremarkable. Physical examination demonstrated that the myodynamia of the right limbs was approximately of grade 5 according to the Lovett Standard (7). A magnetic resonance imaging (MRI) examination showed a sharp cervicomedullary mass extending from the lower medulla to the cervical vertebra C3 level. The mass was hypointense on T1-weighted images (Fig. 1A), compared with the grey or white matter, and hyperintense on T2-weighted images (Fig. 1B). In addition, the mass was enhanced heterogeneously subsequent to contrast agent administration (Fig. 1C). All laboratory tests performed, including blood, urine and coagulation analyses, along with biochemical serum analyses (electrolytes, protein levels, urea, creatinine, glucose, cholesterol and liver enzymes), were found to be normal. In addition, a chest X-ray scan appeared to be normal. Written informed consent was obtained from the patient's family.

The evident clinical symptoms and a definite mass lesion in the cervicomedulla, with a primary diagnosis being glioma of low grade, indicated that surgical excision was required. Following sufficient preoperative preparation, the patient underwent a surgical treatment. Intraoperatively, the medulla and upper cervical cord (between cervical vertebra $\mathrm{C} 1$ and C3) were found to be well-stacked. Immediately after ingression into the spinal cord along the dorsal median sulcus, the tumor mass was detected. The mass had a gray fish-like appearance, moderate blood supply and a clear 

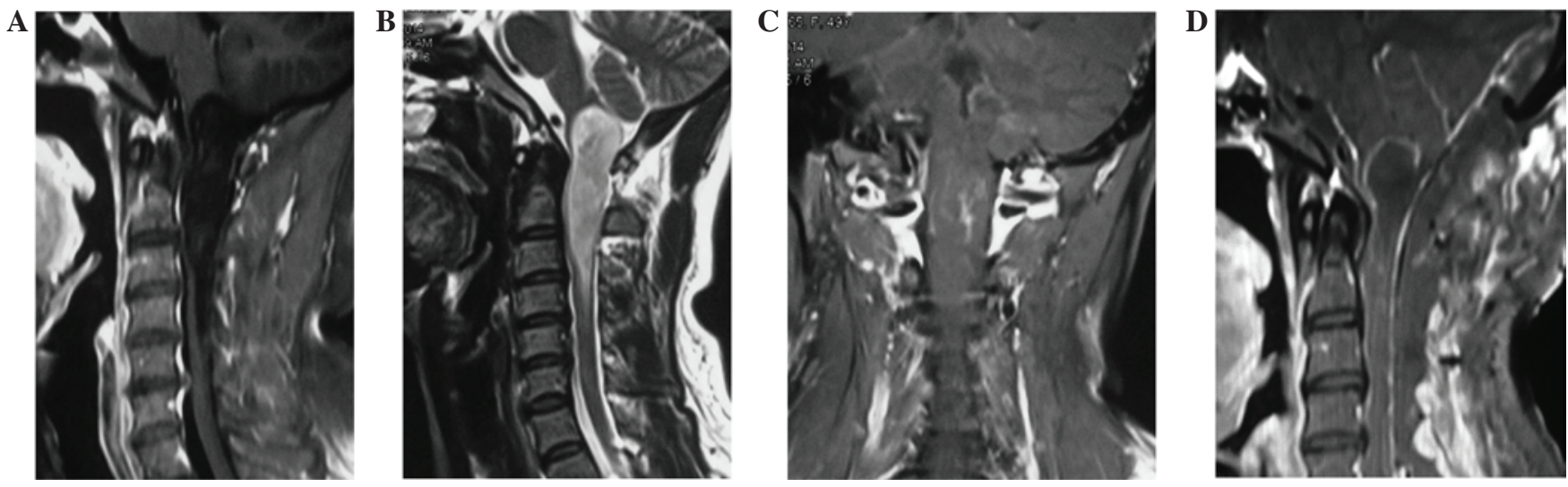

Figure 1. MRI examination revealing a sharp cervicomedullary mass that extended from the lower medulla to the cervical vertebra 3 level. (A) T1-weighted MRI scan showing a hypo-intense lesion and (B) T2-weighted MRI scan showing a hyper-intense lesion. (C) Contrast enhanced MRI scan showing a heterointense lesion. (D) Postoperative MRI scan of the patient showing almost complete excision of the tumor. MRI, magnetic resonance imaging.

A

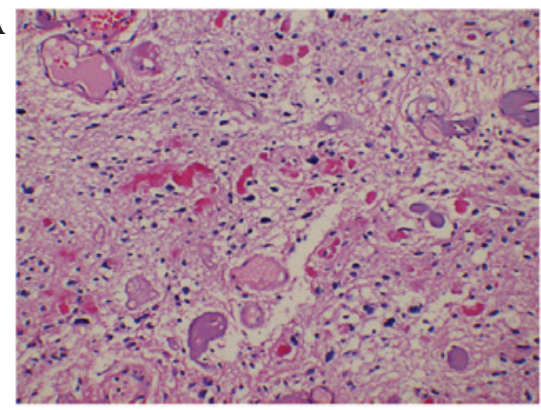

D

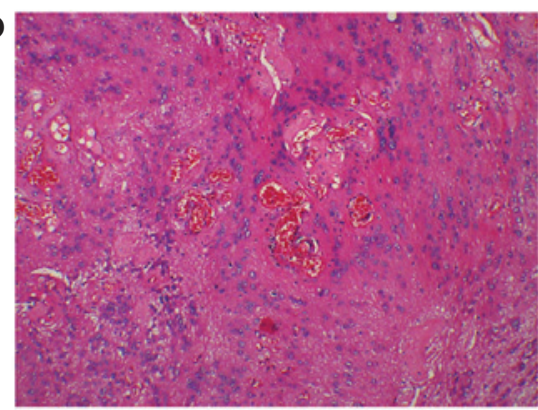

B

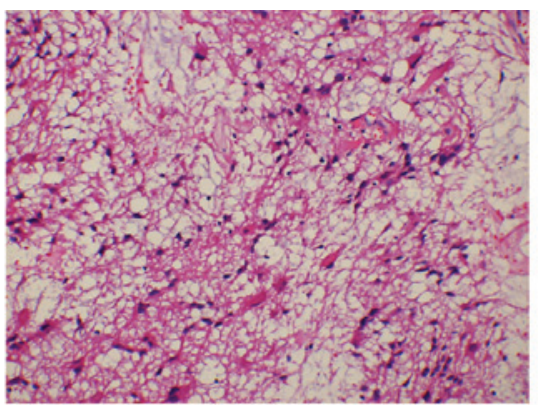

$\mathbf{E}$

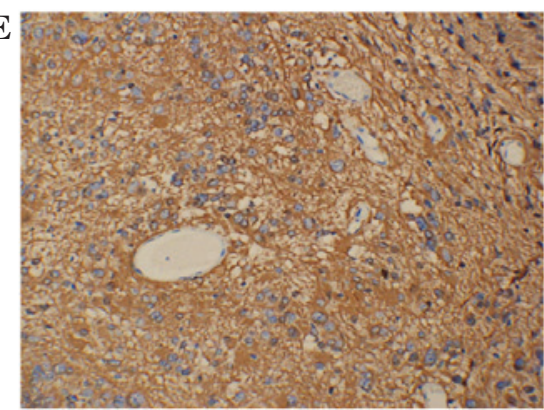

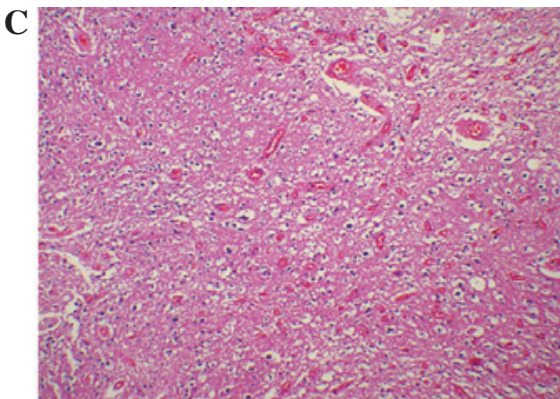

F

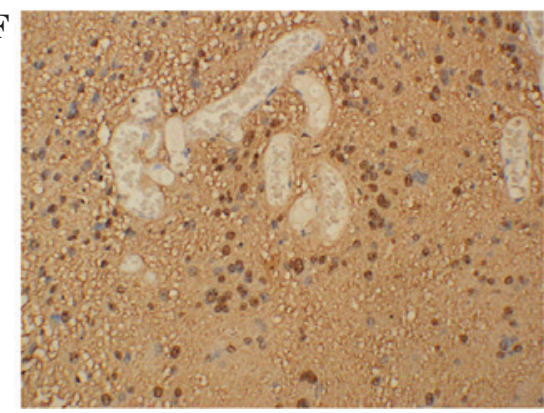

Figure 2. Pathological examination and immunohistochemical staining (hematoxylin and eosin staining; magnification, $\mathrm{x} 200$ ), showing the following features: (A) Rosenthal fibers; (B) loose area; (C) oligodendroglioma-like honeycomb cell area; and (D) braid-like vessels. The tumor cells exhibited positive expression of (E) glial fibrillary acidic protein and (F) S-100 protein.

boundary. After intratumoral decompression, total excision was achieved (Fig. 1D).

Following histopathological examination, the tumor was confirmed to be PA. At high magnification, typical histology characteristics, including Rosenthal fibers, a loose area, an oligodendroglioma-like honeycomb cell area and braid-like vessels, were evident (8). Immunohistochemical staining was performed and the tumor cells were found to be positive for glial fibrillary acidic protein and S-100 protein (Fig. 2).

Postoperatively, the vital signs of the patient were closely monitored, particularly the patient's breathing. The tracheal intubation was removed only after the breathing was confirmed to be stable and vigorous, and the cough reflex was nearly normal ( $24 \mathrm{~h}$ after surgery). Following discharge, the patient did not suffer from any symptoms at the lower cranial nerves. As PA is a benign type of lesion, no postoperative radiotherapy or chemotherapy was required. At 3 months after the opera- tion (August 2014), the myodynamia of the right limbs was comparable to that prior to the operation.

\section{Discussion}

A lesion located in the cervicomedullary junction affects the brainstem, the cervical cord or both regions, depending on its extent and pathology. Involvement of the brainstem is manifested as cranial nerve palsies, decreased respiratory drive and long tract signs, which may result in the tumor being unresectable (8). However, the pathological nature of a cervicomedullary tumor has a great influence on the disease prognosis. For instance, in the case of a PA tumor, complete resection is often curative. Therefore, careful study of preoperative MRI scans is required. In general, the cystic part of PA lesions displays hypointensity on T1-weighted images and hyperintensity in T2-weighted MRI images. In the solid part 
of PA, MRI scans reveal a T1-weighted isointense or slightly hypointense signal, with or without contrast enhancement; by contrast, on T2-weighted scans, the lesions exhibit slight hyperintensity or mixed signals (9). Absence of peritumoral edema is one of the characteristics of PA. Combining these radiological characteristics and the slow onset of the disease, a diagnosis of PA may be considered. Surgical resection remains the first choice of treatment against PA.

In the present case, the tumor mass was carefully removed intraoperatively under a microscope through intratumoral depression, with the least disturbance of the surrounding nerve tissue. During surgery, PA patients are at a high risk of developing bradycardia, and thus monitoring of cardiovascular and respiratory parameters is advocated to detect changes in brainstem function that may result from surgical handling (10). The nucleus tractus solitarius (NTS) and the reticular formation situated in the caudal dorsal medulla are responsible for the cardiac rhythm unsteadiness (11). Even light stimulation of these structures by bipolar forceps during surgery can result in acute hypotension and bradycardia. The middle and caudal regions of the NTS have been reported to represent the sites where afferent nerves mediating cardiovascular and respiratory reflexes make their primary synapse (12). All these regions should be monitored and treated with care during surgery. For patients with a residual tumor, additional management approaches are required, including observation and a combination of surgery, chemotherapy and radiotherapy.

\section{Acknowledgements}

The current research was supported by a grant from the National Natural Science Foundation of China (no. 81101909).

\section{References}

1. Tibbetts KM, Emnett RJ, Gao F, Perry A, Gutmann DH and Leonard JR: Histopathologic predictors of pilocytic astrocytoma event-free survival. Acta Neuropathol 117: 657-665, 2009.

2. Burkhard C, Di Patre PL, Schüler D, Schüler G, Yaşargil MG, Yonekawa Y, Lütolf UM, Kleihues P and Ohgaki H: A population-based study of the incidence and survival rates in patients with pilocytic astrocytoma. J Neurosurg 98: 1170-1174, 2003.

3. Forsyth PA, Shaw EG, Scheithauer BW, O'Fallon JR, Layton DD Jr and Katzmann JA: Supratentorial pilocytic astrocytomas: A clinicopathologic, prognostic, flow cytometric study of 51 patients. Cancer 72: 1335-1342, 1993.

4. Katsetos CD and Krishna L: Lobar pilocytic astrocytomas of the cerebral hemispheres: I. Diagnosis and nosology. Clin Neuropathol 13: 295-305, 1994.

5. Brat DJ and Burger PC: Cerebral pilocytic astrocytoma: Distinction from infiltrating fibrillary astrocytomas. Pathol Case Rev 3: 290-295, 1998.

6. Cyrine S, Sonia Z, Mounir T, Badderedine S, Kalthoum T, Hedi K and Moncef M: Pilocytic astrocytoma: A retrospective study of 32 cases. Clin Neurol Neurosurg 115: 1220-1225, 2012.

7. Dyck PJ, Boes CJ, Mulder D, Millikan C, Windebank AJ, Dyck PJ and Espinosa R: History of standard scoring, notation, and summation of neuromuscular signs. A current survey and recommendation. J Peripher Nerv Syst 10: 158-173, 2005.

8. Nair AP, Mehrotra A, Das KK, Srivastava AK, Sahu RN and Kumar R: Clinico-radiological profile and nuances in the management of cervicomedullary junction intramedullary tumors. Asian J Neurosurg 9: 21-28, 2014.

9. Chourmouzi D, Papadopoulou E, Konstantinidis M, Syrris V, Kouskouras K, Haritani A, Karkavelas G and Dresvelegas A: Manifestations of pilocytic astrocytoma: A pictorial review. Insights Imaging 5: 387-402, 2014.

10. Endo T, Sato K, Takahashi T and Kato M: Acute hypotension and bradycardia by medulla oblongata compression in spinal surgery. J Neurosurg Anesthesiol 13: 310-313, 2001.

11. Lawrence AJ and Jarrott B: Neurochemical modulation of cardiovascular control in the nucleus tractus solitarius. Prog Neurobiol 48: 21-53, 1996.

12. Horiuchi J, Potts PD, Polson JW and Dampney RA: Distribution of neurons projecting to the rostral ventrolateral medullary pressor region that are activated by sustained hypotension. Neuroscience 89: 1319-1329, 1999. 